\title{
Gravity-like Attractions and Fluctuations between Entangled Systems?
}

\author{
Stephane H. Maes ${ }^{1}$
}

June 24, 2020

\begin{abstract}
:
In a multi-fold universe, gravity emerges from Entanglement through the multi-fold mechanisms. As a result, gravity-like effects appear in between entangled particles that they be real or virtual. Long range, massless gravity results from entanglement of massless virtual particles. Entanglement of massive virtual particles leads to massive gravity contributions at very smalls scales. Multi-folds mechanisms also result into a spacetime that is discrete, with a random walk fractal structure and non-commutative geometry that is Lorentz invariant and where spacetime nodes and particles can be modeled with microscopic black holes. All these recover General relativity at large scales and semi-classical model remain valid till smaller scale than usually expected. Gravity can therefore be added to the Standard Model. This can contribute to resolving several open issues with the Standard Model.

All these phenomena result from the observation that attractive gravity-like potentials appear in spacetime between entangled systems, because of the mechanisms proposed in a multi-fold universe to address the EPR paradox. An immediate implication, and opportunity to validate or falsify the model, is that gravity-like effects and fluctuation are predicted to appear between, around or near entangled systems; we just need check if this is encountered in the real world.

This paper discuss situations where attraction due to entanglement, and hence gravity like effects or fluctuations, could be encountered. For example, within or near quantum matter like superconductors or (Bose Einstein Condensates) BECs or within Qubits. One could argue that some indications exist that some of these effects could already have already been observed. We are really seeking falsifiability or validation opportunities for the multifold mechanisms. Early considerations are encouraging.
\end{abstract}

Discussing some related experiments led us to also address how shielding is correctly modeled with multi-fold mechanisms: Faraday cages do not weaken gravity!

\section{Introduction}

The new preprint [1] proposes contributions to several open problems in physics like the reconciliation of General Relativity with Quantum Physics, explaining the origin of gravity proposed as emerging from quantum (EPREinstein Podolsky Rosen) entanglement between particles [5], detailing contributions to dark matter and dark energy and explaining other Standard Model mysteries without requiring New Physics beyond the Standard Model other than the addition of gravity to the Standard Model Lagrangian. All this is achieved in a multi-fold universe that may well model our real universe, which remains to be validated.

With the proposed model of [1], spacetime and Physics are modeled from Planck scales to quantum and macroscopic scales and semi classical approaches appear valid till very small scales. In [1], it is argued that spacetime is discrete, with a random walk-based fractal structure, fractional and noncommutative at, and above Planck scales (with a 2-D behavior and Lorentz invariance preserved by random walks till the early moments of the

\footnotetext{
${ }^{1}$ shmaes.physics@gmail.com
} 
universe). Spacetime results from past random walks of particles. Spacetime locations and particles can be modeled as microscopic black holes (Schwarzschild for photons and spacetime coordinates, and metrics between Reisner Nordstrom [2] and Kerr Newman [3] for massive and possibly charged particles - the latter being possibly extremal). Although surprising, [1] recovers results consistent with other like [4], while also being able to justify the initial assumptions of black holes from the gravity or entanglement model. The resulting gravity model recovers General Relativity (GR) at larger scale, as a 4-D process, with massless gravity, but also with massive gravity components at very small scale that make gravity significant these scales. Semi-classical models also work well till way smaller scales than usually expected.

In the present paper, we remain at a high level of analysis. It makes the points accessible to a wider audience and keeps the door open to further papers or discussions devoted to details of interest. Yet, it requires the reader to review [1], as we do not revisit here all the details of the multi-fold mechanism or reconstruction of spacetime. The followings subsections are organized as a series of observations in [1] where gravity like effects are expected to result from entanglement and should be observable, at least indirectly through some resulting effects. Direct observation will remain challenging because of the expected weakness of the attractions. Our analysis is by no means exhaustive. However, we hope that it will intrigue enough the reader to push him or her to dig deeper. Most of the more detailed (or entry point) references are provided in [1], and so every statement is not motivated here or presented with the most appropriate references. This paper is rather a story tale. "[1]" appears often, as a person or a model, to refer to the original arguments, analysis, mechanisms or proposals discussed in [1].

\section{Entanglement effects in Multi-fold universes}

The mechanisms of multi-folds, the main feature proposed in [1], trigger activation of additional structures (folds) when particles are (EPR) entangled so that additional paths can traverse the folds, where the EPR entangled particles can always meet as a same exit points. Doing so, all the activated folds (i.e. multi-folds) create attractive potentials in $\frac{1}{r}$ in between the entangled particles $\left(\frac{1}{r^{2}}\right.$ per fold). The attraction is towards their source or center the mass, depending of the use cases and movements (and masses involved - entangled particles can be massive or massless). When involving virtual particles emitted by a source of energy, this potential is reminiscent of gravity and [1] attributes gravity to these effects. It can also be looked as adding contributions of the Ricci curvature scalar R of the folds, from all matter or energy contributions, to build a new Ricci curvature scalar field R and, with the direction of attraction information, a new consistent Ricci curvature tensor. Doing so, for all sources of energy, recovers Einstein's GR field equations (or Hilbert Einstein Action); which is amazing as invariance of surfaces (the real geometrical meaning behind the Hilbert Einstein Action) or variants of the Hilbert Einstein have, at no point, be postulated in [1] prior to that determination (something that can't exactly be said the same way for strings). Also, the multi-folds have a spin-2 symmetry.

So, it is predicted in [1], that (EPR) entanglement between particles (or larger systems), results into attractive potentials in $\frac{1}{r}$ towards the center of mass, with $r$ the distance between form the center of mass, in between the entangled particles (on the support domain of the mapping), if integration takes place over $r$. That is over a system of entangled particles or for the range of uncertainty. Otherwise, each particles contribute a $\frac{1}{r^{2}}$ per fold contribution. For gravity, the integration of $r$ goes to infinity, hence the generic gravity like statement.

It is also important to note for completeness that [1] postulates that such effects only exist when entanglement is the result of interaction occurring locally (same source location). Other situations are considered as hierarchical and thought not to contribute an additional effective potential. Yet, as in force composition, the different parts involved in a hierarchical event also amount to attractive effects; so attraction exist but as force composition. Also, if the entanglement is the effect of many repeated interactions (e.g. electron to phonon to electron), while hierarchical, the effects with composition will just appear as a normal non-hierarchical effect with attractive potential (at least in first approximation). So solid state entanglements a la superconductors for examples are 
modeled as nonhierarchical entanglement in this discussion; even if, in reality, it is the outcome of complex hierarchical composition of attractive potentials.

\section{Gravity like fluctuations near (in between) entangled systems}

An immediate consequence of the mechanism and model proposed in [1], is that fluctuations of gravity-like effects (in $\frac{1}{r}$ - when macroscopic and in $\frac{1}{r^{2}}$ when mostly between localized individual particles. These effects are very small (as is gravity beyond very small scales), so direct observation is probably hopeless for the near future, if ever. We will need clever indirect ways or macroscopic additive effects to be able to validate our model.

A non-exhaustive list of candidate scenarios where such gravity like fluctuations are predicted to exist is provided here:

- Gravity like effects or fluctuations within, and in proximity of superconductors. Superconductors involve of combinations of Bardeen Cooper Schrieffer (BCS) pairs (at low temperatures and for low temperature superconductors) [7] and Bose Einstein Condensate (BEC) pairs [8] (after a transition from BCS pairs for high temperature superconductors) as well BEC pairs of pairs etc. in high temperature superconductors [6]. According to the mechanisms described in [1]:

- Attraction should occur within the bulk of the superconductors. It should also be with stronger effects for high temperature superconductors, because BEC pairs are smaller than BCS pairs (That spread all over the material over many crystal cells).

- This kind of effects have been anecdotally reported (see [9] for one of the most recent compilation of these controversial and hard to reproduce experiments) ${ }^{2}$. However, we urge the reader to be cautious in reading beyond the descriptions of the experiments and results and the references as we do not necessarily subscribe with the presentation of the experiments as accepted facts or many aspects of the proposed explanations or assertions in some of the listed references material, of anti-gravity, gravity shielding or repulsive gravity effects and other families or properties of gravitons-like particles. Unfortunately, the results experiments seem to have never been rigorously confirmed or unambiguously analyzed.

- In our view, these reported effects, if corroborated, and if we understand well the setup of the two experiments, could result from super-conductor internal stress within the electromagnetic field (between separated BEC BCS-pairs) plus vacuum polarizations. The latter results from entanglement attractions between the produced polarized virtual pairs. When the discharges occur, the superconductor and the vacuum polarization relaxes and so does the vacuum entanglement and attraction potential, resulting into a gravity fluctuation or wave that propagate at the same speed as the polarization relaxation. The relaxation produce a "expansion effects", wherever polarization was present in the vacuum as well as within the superconductor and could explain the effects on the emitter or on the test masses. It would appear as an initially repulsive effect as the relaxation wave propagates. This explanation to these controversial experiments have never been proposed in the related literature as summarized in [9]. The complications of the shields is discussed in Appendix A.

\footnotetext{
${ }^{2}$ We are cautious about citing and concerned about the extensive discussion presented here. Indeed the experiment result mentioned here are seen as controversial. We mention them, more as examples of indirect ways to experiments with effects predicted by [1], than as successfully reviewed experimental results that we would want to rely on.
} 
- If true (both the observations and our suggested explanation), then we have a resounding indirect confirmation of the mechanisms described (attraction due to entanglement) in [1]; not just for entanglements within the superconductor but also the entanglement of the polarized vacuum.

- The stronger attraction within the high temperature superconductor creates a stronger effect than with low temperature superconductor material when the pairs are pushed to its boundaries by the electromagnetic field. A nonentangled material only see the vacuum effect. Without superconductors, i.e. in normal discharge situations, only vacuum polarization relaxation takes place. This is not sufficient. The fact that recoil may be better corroborated while radiation effects seems (often) no reproducible could come from the fact that the relaxation effect within the superconductor always takes place and is stronger than vacuum polarization relaxation. The other case (figure 1-a in [9]) requires suitable polarization beyond the right electrodes till the test mass something and it is a much weaker effect.

- Superconductors are also involved in these experiments also because of their known propensity of quantum matter like superconductors to amplify or reflect the vacuum polarization effects; something well known since the work for example of deWitt [10] and also involved in the still unconfirmed gravitational Casimir effect proposal [11]. These works predict effects of gravity on superconductor, not gravity like effect produce by super conductors. The distinction matters and shows the challenge in distinguishing the two types of effects if we want to validate the gravity like attraction generated by entanglement.

- To be convincing, we should see larger effects than expected by just contributions à la [10]. The results, with the problems already mentioned seem to indicate that it may be the case.

- As another related potential corroboration, building on the ideas of [10], it has also been proposed that an effect for gravitation analogous to the London moment in superconductor could exist for gravitons, in rotating superconductors, in a varying strong magnetic field [12]. Again, the magnetic field would push BEC BCS-pairs towards the surface of the superconductor and, as a result, bring stronger gravitation effect leaks observable outside and very near the super conductor, where a frame dragging effect as in GR, but stronger could be observed. Such effects have been observed [12]. However, the reported results were again in our view not clear enough to assess for sure if they would match our frame dragging expectation. It seems that they might.

- It is also important to understand all aspects of the experiments and details are missing on the actual results and in particular make sure that the effect are due to entanglement and not a variation a la [10], where frame dragging would be explained solely by the rotation flipping the roles (here the super conductor rotates, the detector is fixed) without the contributions of the attraction / gravity like fluctuation due to entanglement.

- The effect must be larger than normal frame dragging (undetectable) or effects explained by [10]. More work to model how [10] impacts the experimentation and if we can really detect an unexpected additional effect. Assuming that [12] did correctly account for [10], then according to the result, they have unaccounted for effects.

- The proposed setup of [12] and variations could be good ways (better than the first set of discharge experiments) to (indirectly) validate the multi-fold mechanisms. However, we would prefer experiments that are not involving and mixing other Physics (like strong magnetic fields, strong electromagnetic pulses etc.) to avoid the risk of misinterpretations and combinations of all these effects from superconductor, existing 
gravity and electromagnetism interactions. Electromagnetic fields were required because London - Meissner types of behaviors can amplify our predicted attraction . Unfortunately, we could not determine based on the research reports what of the side effects of the fields, as discussed here, have been accounted for in the results.

- Quantum matter, like BECs, superfluids, supermetals etc. are other candidates. The gravity fluctuation effects to look for are similar to what is discussed above for superconductors. The particular existing results discussed above for superconductor may not be repeatable or may need adaptation depending on the type of quantum material.

- Quark Gluon Plasma (QGP) is another example of BEC [14]. Here, we see two avenue for confirmations:

○ Experimentally when such plasma are formed in high energy accelerators [13]. It would be worth looking if any perturbations due to attractive potentials could be modeled and observed

- Theoretical models of cosmology (early moments after the big bang) and stellar physics could consider if adding such considerations could introduce new prediction or effects when involving large quantities of plasma and thus entanglement. The main reason being that at the scale of the universe or of stars, even small effects can start to play meaningful roles.

- Speaking of which, $[1,5]$ showed of an effect associated to entanglement can qualitatively explain the dark matter effects, without requiring New Physics. It seems also consistent with the observations of galaxies that seem not to contain dark matter; something that most other models have had difficulties to handle. This is quite a potential confirmation, but we now need to proceed towards a more quantitative model of [1] so that we can determine if the number match to account for dark matter (or a portion of it).

- Validating [5] would be of great interest. It would after all, with the conclusions of our model, probably and most influential entanglement effect that we can think of (short of large or even larger, scale spacetime entanglement, proposed by others, but not something that we support).

- It is certainly encouraging that in addition, $[1,15]$ can also explains effects that contribute to cosmological inflation and dark energy as well as a small cosmological constant that does not conflict with the QFT vacuum energy density estimates.

- Qubits are entangled systems achieved by different mechanisms like trapped ions, superconductors etc. [16]. They are at the code of quantum computing and larger Qubit systems are being built as time passes. These are not yet large enough for our needs, but things may change rapidly. Within the Qubits, if measurable, attraction would be a sign of entanglement and therefore a way to detect entanglement without observing it; something forbidden by the non-observability of entanglement [17]. Being able to do so would be a great tool for quantum computing and validation of our predictions.

- For quantum computing, teleportation or other purpose, researchers are entangling bigger systems like atoms, larger and larger molecules, wider atom systems or even biological systems; all involving huge amounts of entities (see for example [18-20]). The bigger these systems are the better are the chance to directly or indirectly determine if gravity fluctuations appear among them, as long that we do not hit the snag of hierarchical entanglement not producing attractive potentials. So some precaution are needed to understand if validation is possible or if the absence of attraction would implies falsifiability of our model or rather such the dominance of hierarchical entanglement effects.

\section{Other effects and Considerations}

It is also worth also noting that [1] predicts impact of the multi-folds effects on the Standard Model. So far, we have used that explain some open problems with the standard model, without requiring new physics. We have shown how entanglement would also appear; but we have not yet found any situation (besides dark matter as in [5]) where it is the contributing factor, versus rather the massive gravity contribution term at small scales also predicted by [1] and expected to be non-negligible at small scales. So far it is that latter mechanism that is invoked in [1] to contribute explanations. See [21] for a list of papers derived from [1], many discussing the impact on the standard model or on New Physics beyond the Standard Model. 
That is not to say that, even if possibly surprising, the model proposed in [1] is in fact already contained in many existing conventional physics as well as New Physics around Superstrings and the AdS/CFT correspondence conjecture [22]. Indeed, see for example [23-24] showing how entanglement and spacetime curvature relate. See $[1,22]$ for analysis of how our model also relates to superstring and more directly on topic, how the ER=EPR conjecture [25] is very much a more limited model corroborating the multi-fold mechanisms (see for example [26]); but missing the resulting impact of gravity like potentials towards the center of mass. Non-transferability of the wormholes and misreading of the curvature implications of the entangled black holes may possibly be why these models have not (yet) reached our conclusions. For us, the beauty is that we do not need the New Physics, we just need to add gravity (string enough at smalls scales) to the Standard Model. There is enough material to start making a case for this [21].

\section{Conclusions}

In this paper, we have compiled examples of situation where it might be possible to observe gravity like fluctuations due to entanglement, as predicted by the multi-fold mechanisms proposed in [1].

At this stage, we hope to find more experiments, effects or model where the additional gravity fluctuation due to entanglement plays a significant role that makes it or its consequence detectable. It is essential to the validation or falsifiability of the multi-fold mechanism proposed in [1]. Doing so if for future work but we can only encourage any such experiments or to keep our predictions in mind quantum matter or quantum computing and teleportation experiments, just in case.

A few challenges remain. The main one being that just like for gravity, at the scale considered, the effects are so small that it will be very hard to detect them, especially directly. Yet our proposal for dark matter already shows that there are ways and there is hope. We also have high hopes for superconductors and BEC experiments. We already pointed out to anecdotal that may corroborate; even if not necessarily as the authors of these experiments would have expected.

Of course, another challenge is that the model of [1] is more qualitative than quantitative. Now, it is a priority for us to evolve towards more quantitative approaches by evolving form proportionality equation to the real coupling factors and estimate these factors (e.g. by relating to expected values in classical situations). We aim with future work to get such better quantitative predictions as well as to evangelize experimentations base don the present paper. Not being currently active in a Physics institution, currently limits our ability to directly attempt an experimental program ourselves.

Our hope with this publication is that others will get ideas on how to validate our model directly or indirectly. We certainly welcome such, or any other, collaborations.

Needless to say that the early hints of corroboration presented here, the contributions to addressing open issues covered in $[1,21]$ and the fact that Physics all along maybe hinted at the multi-folds mechanism, are strong encouragements. We hope it will convince the community to spend some cycle on what [1] proposes.

Note (10/2/20): The progresses towards larger entangled systems reported recently in $[27,28]$, as well as [18-20], will hopefully result into some focused efforts to test our model of attractive gravity like effects between and among entangled systems.

References: (most references come from popular science to make the discussion more approachable) 
[1]: Stephane H. Maes, (2020) “Quantum Gravity Emergence from Entanglement in a Multi-Fold

Universe", viXra:2006.0088v1, (June 9, 2020).

[2]: https://en.wikipedia.org/wiki/Reissner\%E2\%80\%93Nordstr\%C3\%B6m_metric

[3]: https://en.wikipedia.org/wiki/Kerr-Newman metric

[4]: Burinskii, Alexander, (2008), "The Dirac-Kerr-Newman electron", arXiv:0507109v4

[5]: https://en.wikipedia.org/wiki/EPR_paradox

[6]: https://en.wikipedia.org/wiki/Superconductivity

[7]: https://en.wikipedia.org/wiki/BCS theory

[8]: https://en.wikipedia.org/wiki/Bose\%E2\%80\%93Einstein condensate

[9]: Giovanni Modanese, (2014), "Gravity-Superconductors Interactions as a Possible Means to Exchange

Momentum with the Vacuum", arXiv:1408.1636v1

[10]: Bryce S. DeWitt, (1966), "Superconductors and Gravitational Drag", Phys. Rev. Lett. 16, 1092

[11]: James Q. Quach, (2015), "Gravitational Casimir effect", arXiv:1502.07429v1

[12]: Clovis Jacinto de Matos, Martin Tajmar (2006). "Gravitomagnetic London Moment and the Graviton Mass

inside a Superconductor", arXiv:cond-mat/0602591

[13]: ALICE Collaboration, (2018), "Anisotropic flow in Xe-Xe collisions at sqrt\{s_\{NN\}\}=5.44 TeV",

arXiv:1805.01832v2

[14]: https://en.wikipedia.org/wiki/Quark\%E2\%80\%93gluon_plasma

[15]: Stephane H Maes, (2020), "Explaining Dark Energy, Small Cosmological Constant and Inflation Without New

Physics?", viXra:2006.0261v1, https://shmaesphysics.wordpress.com/2020/06/19/explaining-dark-energy-smallcosmological-constant-and-inflation-without-new-physics/, June 19, 2020.

[16]: https://en.wikipedia.org/wiki/Qubit

[17]: Ning Bao and Jason Pollack and Grant N. Remmen, (2015), "Wormhole and entanglement (non-)detection in the ER=EPR correspondence", arXiv:1509.05426

[18]: C. F. Ockeloen-Korppi, E. Damskagg, J.-M. Pirkkalainen, A. A. Clerk, F. Massel, M. J. Woolley, M. A. Sillanpaa, (2017), "Entangled massive mechanical oscillators", arXiv:1711.01640v1

[19]: Yaakov Y. Fein et al. (2019), "Quantum superposition of molecules beyond 25 kDa", Nature Physicss.

[20]: Kong, J., Jiménez-Martínez, R., Troullinou, C. et al., (2020), "Measurement-induced, spatially-extended entanglement in a hot, strongly-interacting atomic system". Nat Commun 11, 2415.

[21]: https://shmaesphysics.wordpress.com/shmaes-physics-site-navigation/

[22]: Stephane H Maes, (2020), “Dualities or Analogies between Superstrings and Multi-fold

Universe", viXra:2006.0178v1, https://shmaesphysics.wordpress.com/2020/06/14/dualities-or-analogies-betweensuperstrings-and-multi-fold-universes/, June 14, 2020.

[23]: ChunJun Cao, Sean M. Carroll, Spyridon Michalakis, (2016). "Space from Hilbert Space: Recovering Geometry from Bulk Entanglement", arXiv:1606.08444v3.

[24]: van Raamsdonk, Mark (2010). "Building up spacetime with quantum entanglement", Gen. Rel. Grav. 42 (14):

2323-2329. arXiv:1005.3035

[25]: https://en.wikipedia.org/wiki/ER\%3DEPR

[26]: Julian Sonner, (2013), "Holographic Schwinger Effect and the Geometry of Entanglement", arXiv:1307.6850v3.

[27]: https://www.sciencealert.com/physicists-push-the-limits-on-quantum-mechanics-entangling-two-very-

different-objects

[28]: Rodrigo A. Thomas, Michał Parniak, Christoffer $\varnothing$ stfeldt, Chistoffer B. Møller, Christian Bærentsen, Yeghishe Tsaturyan, Albert Schliesser, Jürgen Appel, Emil Zeuthen, Eugene S. Polzik, (2020), "Entanglement between Distant Macroscopic Mechanical and Spin Systems", arXiv:2003.11310v1

\section{Appendix A - No gravity shields in Multi-fold Universes}

In [9], the experiences of figure 1 and 2, sensors are described as positioned in shielded boxes or behind shield screens, we do interpret this as electromagnetic shields (as faraday cages or large screens). This is certainly 
challenging a direct vacuum polarization story beyond the shield. We did not want to bring this up in the main discussion and add more controversies.

Obviously, gravity screens do not exist. [1] must be able to account for no weakening of gravity within Faraday cages for example, despite our mechanisms relying on virtual particles. If only virtual neutrinos were to contribute, gravity would be weakened within such a cage, which is obviously not the case. In general for the multi-fold mechanisms of [1], when the virtual particles tries to reach a test particle within an electromagnetic shield, it does affect the four -vector potential of the particles on shield. Considering the system shield + target particle, its total energy is affected and it affects the energy source available to multi-folds affecting the test particle. The combined effect is hierarchical and the composition appears as if the effect went through the shield: The mass of the shield particle is increased to reflect the potential from the source. The outcome is the same as if no shield were present except form the additional proper gravitational effect of the shield as source.

A dedicated upcoming paper or an update of [1] will explicitly address these shielding concerns with the multi-fold mechanisms.

Coming back to [9], our plausible explanation stops at the shield. So what could be happening next? The gravity fluctuation due to the relaxation of the vacuum polarization (e.g. in figure 2 of [9]) affects the 4-vector potential as a fluctuation that therefore could continue beyond the shield as a gravity fluctuation. Remember, we only try to interpret [9] at the light of [1]. We are in no position to corroborate what actually was observed. 